$\begin{array}{llllllllllllll}A & R & T & I & C & U & L & O\end{array}$

$D E \quad R E F L E X X I O ́ O$

\title{
LA ORGANIZACIÓN EMPRESARIAL ASOCIATIVA COMO ESTRATEGIA PARA LA INCLUSIÓN PRODUCTIVA DE LAS PEQUEÑAS Y MEDIANAS EMPRESAS
}

\author{
THE ASSOCIATIVE BUSINESS ORGANIZATION AS A STRATEGY FOR THE \\ PRODUCTIVE INCLUSION OF THE SMALL AND MÉDIUM BUSINESS
}

\author{
Por: Kenia Victoria Cogollo* \\ Rosaura Arrieta Flórez ${ }^{* *}$ \\ Recibido: 30 de junio de 2013. Aprobado. 23 de marzo 2014.
}

Candidata a doctor en Ciencias Sociales y Trabajo Social: Desigualdades e Intervención Social. Docente programa de sidad de Cartagena.

**Magíster en Política Social. Docente investigadora del Instituto de Poĺticas Públicas, Regionol $y$ de Gobiern, versidad de Cartagena.

\section{RESUMEN:}

Este artículo presenta en primer lugar una lectura de los indicadores socioeconómicos de la ciudad, que permitan argumentar la hipótesis de la importancia de nuevas formas de organización asociativa como alternativa para lograr un desarrollo económico inclusivo. Posteriormente se hace referencia a algunos elementos de la primera experiencia asociativa en la ciudad y que marcó un hito en cuanto a procesos organizativos en el sector metalmecánico de la pequeña y mediana empresa; y a partir de allí se reflexiona sobre la importancia de articular procesos formativos de asociatividad a la formulación, promoción y consolidación de una política de inclusión productiva, que impacte realmente en la dinámica económica y el contexto local.

Palabras claves: Asociatividad, desarrollo local, Inclusión productiva, pequeña y mediana empresa, política social.

\begin{abstract}
:
This article presents in first place a reading of the socio-economic indicators of the city, which allow to argue the hypothesis of the importance of new forms of associative organization as an alternative to reach an inclusive economic development. Subsequently we refer to some elements of the first associative experience in the city and which marked a milestone in terms of organizational processes in the metal-mechanic of the small and medium enterprises, and from there we reflect about the importance of articulation of formative processes of associativity to the formulation, promotion and consolidation of an inclusive productive policy, which really impacts in the economic dynamic and the local context.
\end{abstract}

Key Words: Association, small and medium business, local development, social policy.

\section{Introducción}

E n Cartagena de Indias, los crecientes niveles de desigualdad social y la brecha de la pobreza hacen pensar seriamente que se requiere presentar alternativas que configuren un acuerdo social entre los diferentes actores del desarrollo local. Este trabajo centra su análisis en la asociatividad como estrategia para la inclusión productiva de las pequeñas y medianas y empresas y con ella el desarrollo de la ciudad y sus habitantes. 


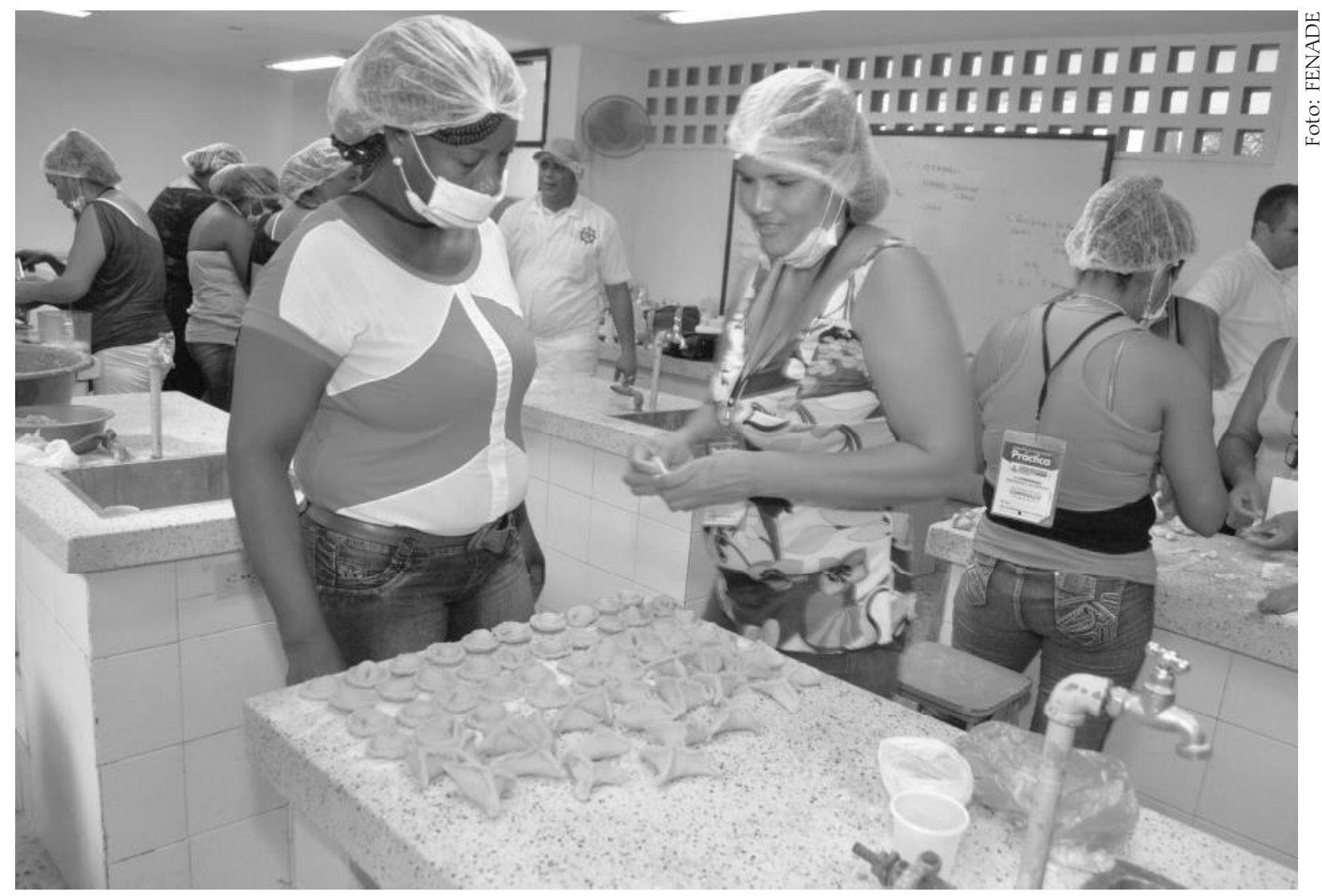

En palabras de Vázquez-Barquero:

"Un proceso de crecimiento económico y de cambio estructural que conduce a una mejora en el nivel de vida de la población local, en el que se pueden identificar tres dimensiones: la dimensión económica, en la que los empresarios locales usan su capacidad para organizar los factores productivos locales con niveles de productividad suficientes para ser competitivos en los mercados; la dimensión sociocultural, en que los valores y las instituciones sirven de base al proceso de desarrollo; y, finalmente, una dimensión político-administrativa en que las políticas territoriales permiten crear un entorno económico local favorable, protegerlo de interferencias externas e impulsar el desarrollo local ${ }^{1 \prime \prime}$.

Este artículo presenta en primer lugar una lectura de los indicadores socioeconómicos de la ciudad, que permitan argumentar la hipótesis de la importancia de nuevas formas de organización asociativa como alternativa para

'Vázquez, Barquero cal una estrategia de creación estrategia de Madrid: Pirámide. 
${ }^{2}$ Castells, M (2001). La ciudad de la nueva economía. Papeles de población, enero-marzo, (27) $207-221$

${ }^{3}$ Corredor, C (2010). La política social en clave de derechos. Bogotá: Universidad Nacional de Colombia. lograr un desarrollo económico inclusivo. Posteriormente se hace referencia a algunos elementos de la primera experiencia asociativa en la ciudad y que marcó un hito en cuanto a procesos organizativos en el sector metalmecánico de la pequeña y mediana empresa; y a partir de allí se reflexiona sobre la importancia de articular procesos formativos de asociatividad a la formulación, promoción y consolidación de una política de inclusión productiva, que impacte realmente en la dinámica económica y el contexto local.

Manuel Castells propone a las ciudades como "... claves tanto como productoras de procesos de generación de riquezas, como de la capacidad social de corregir los efectos desintegradores y destructores de una economía de redes sin ninguna referencia a valores sociales más amplios, mas colectivos, o no medibles en el mercado2." En este sentido la articulación de la política económica y la política social se convierte en determinante del logro de ciudades más modernas, competitivas y equitativas, que posibiliten el desarrollo humano integral.

En este sentido, y a pesar que la escasa incidencia de los territorios en la definición de la política macroeconómica y laboral es mínima, los gobiernos locales cuentan con algunos instrumentos de política económica y social que unidos a las potencialidades propias del territorio pueden generar procesos de inclusión económica y ampliación de oportunidades para todos. Uno de estas estrategias es el fomento de formas asociativas de trabajo, las cuales permiten aprovechar las iniciativas económicas locales y vincular a mayor parte de la población, especialmente la que se encuentra en mayor situación de pobreza y vulnerabilidad ${ }^{3}$.

\section{I.Contextualización}

- egún el Departamento Administrativo Nacional de Estadísticas (DANE) en Colombia el 99.1\% de las empresas (más de medio millón) es catalogada como pequeña y mediana; lo que las convierte en las mayores generadoras de empleos directos e indirectos, y a la vez en mayores generadoras de exportaciones no tradicionales. No obstante, aunque estas son intensivas en mano de obra, su estructura de capital es baja y al compararlas con las grandes empresas son poco competitivas por carecer de inversión en tecnología de punta y carencias en capital.

Para determinar las potencialidades y debilidades que tiene Cartagena para lograr una relación armónica entre la política económica y social que permita 
Gráfico 1. Pirámide poblacional 1985

Gráfico 2. Pirámide poblacional 2010

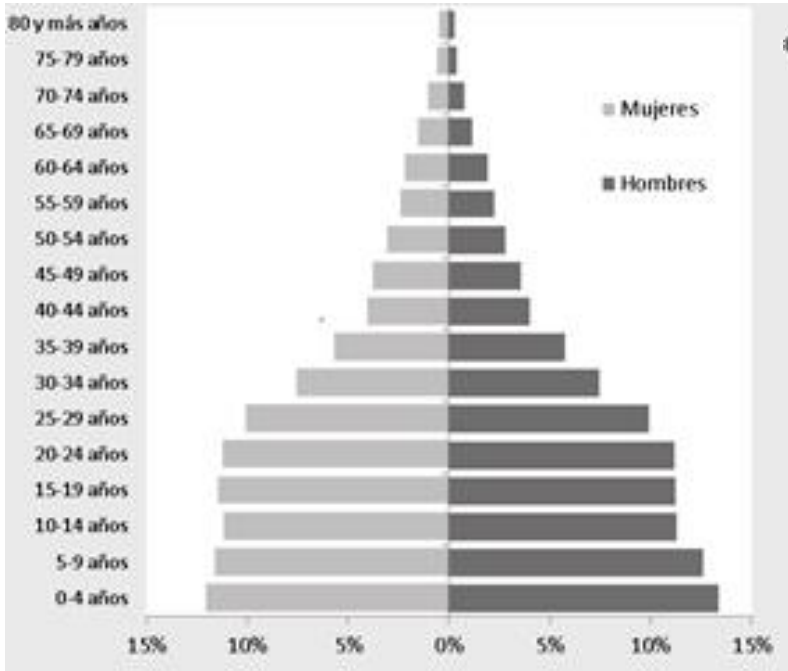

Fuente: Dane. Proyecciones Censo 2005

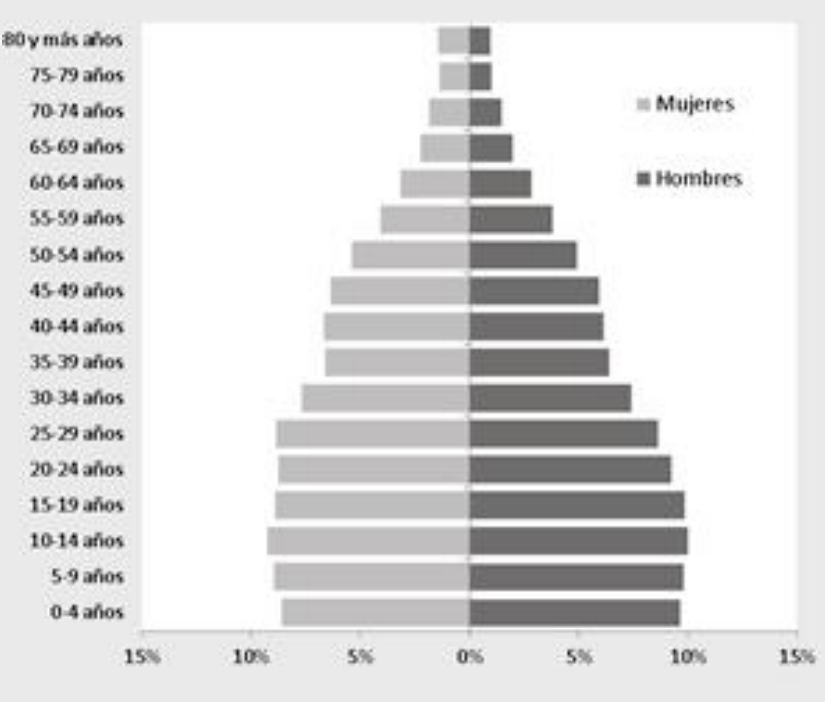

Fuente: Las autoras con base en Dane.

alcanzar crecimiento con equidad, es necesario analizar el territorio con enfoque poblacional; es decir, reconociendo a la población como entidad activa en constante transformación, que demanda bienes y servicios, que es sujeto de derechos y responsabilidades y gestora de su propio proceso de desarrollo dentro de una perspectiva necesariamente más amplia, la del desarrollo integral.

En este sentido, en las últimas décadas se pueden identificar en la ciudad diversas tendencias que inciden en los logros y las posibilidades del desarrollo humano. La primera es la rápida expansión poblacional que experimenta Cartagena desde la segunda mitad del siglo XX, induciendo a su vez a cambios demográficos que han elevado sostenidamente la demanda por servicios sociales de las personas. Estos cambios resultan de dos procesos: por una parte, del angostamiento de la pirámide poblacional por la dinámica connatural de envejecimiento de la población, y por otra, los resultados del desplazamiento forzado que cuenta a Cartagena como el principal receptor de población en esta situación en el departamento de Bolívar desde mediados de los años noventa ${ }^{4}$.

A esto se le suma que más de la mitad de la población en edad de trabajar no supera los treinta años. Es decir, existe un alto potencial de mano de obra presente y futura (bono poblacional) que presiona el mercado laboral al tiempo que existe una fuerte presión por la demanda de servicios sociales como salud y educación. (Ver gráfico1)

De estos hechos demográficos se desprende una segunda tendencia: los efectos ${ }^{4}$ Según la consultoría
para los Derechos Humanos y el Desplazamiento (Codhes) más de setenta mil familias desplazadas llegaron a la ciudad hasta 2010.

Palobra No. 14. Agosto de 2014 
Gráfico 3. Pirámide poblacional Red Juntos 2010

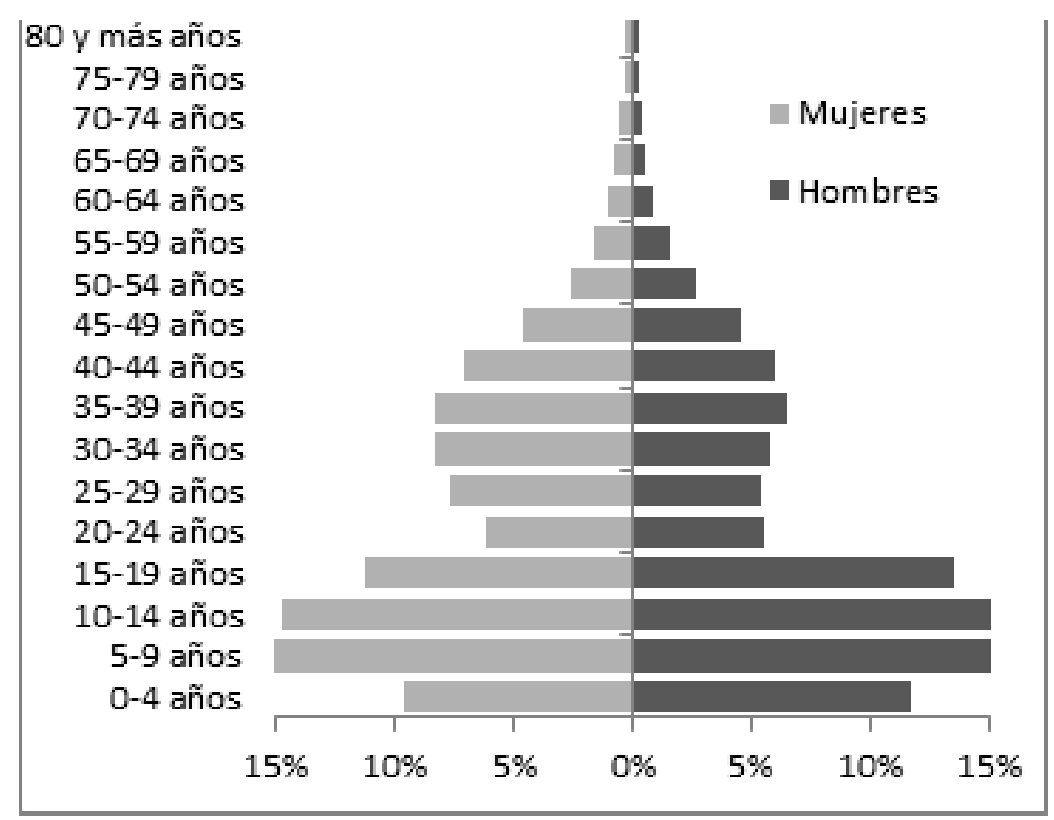

Fuente: Secretaria de Hacienda Distrital con base en Sisben III. del cambio de la estructura poblacional sobre el mercado de trabajo. La concentración en el área urbana y la mayoría de edad de la población bolivarense impone desafíos a la economía local para absorber la creciente población en edad de trabajar de Cartagena, que adquiere mayor peso relativo en los próximos años.

La pregunta obligada es entonces: ¿Qué le ofrece esta fuerza laboral a la ciudad? ¿Cuáles son sus capacidades? ¿Cómo participa del mercado laboral de la ciudad? Para responder estas preguntas se comparó la pirámide censal para el año 2010 con la de la estrategia Unidos ${ }^{5}$. Se observa que la pirámide poblacional de la población en pobreza extrema es más ancha en la base y en la cima, lo que indica el reto mayor de la política social del Distrito en la medida en que es necesario ofrecer una atención integral a la base de la pirámide para romper las trampas de la pobreza; pero también hay que ofrecer posibilidades de absorción de esta gran fuerza laboral que tiene unas características y dotaciones especiales. (Ver gráfico 3).

Según Rueda y Espinosa ${ }^{6}$, una de las características de la economía de la ciudad en los últimos años es la poca respuesta de la tasa de desempleo a los cambios en la situación económica. Si bien el número de empleos ha aumentado, la tasa de desempleo ha permanecido en niveles relativamente elevados. Es decir, a pesar de la dinámica económica de la ciudad se generan menos empleos

${ }^{5} \mathrm{La}$ estrategia Unidos es un instrumento de focavicios sociales del Esterbeneficien de mano beneficien de manera la población más pobre y vulnerable.

${ }^{6}$ Rueda y Espinosa hoy serán los pobres de mañana? Determinantes de la pobreza y la vulnerabilidad en Cartagena de Indias. Documentos de trabajo. (11). que en el resto del país; entre otras razones porque el aumento de los puestos de trabajo creados es menor al crecimiento vegetativo de la población. En Cartagena el aumento del empleo ha sido generado por el sector informal, es decir, la misma gente crea oportunidades de trabajo, generalmente por fuera del sistema de protección social y no en el sector productivo. En los últimos meses, el empleo de calidad ha bajado, mientras aumentan los llamados trabajadores por cuenta propia. 


\section{Mercado laboral y pobreza}

La literatura ha demostrado que el crecimiento económico es indispensable para la reducción de la pobreza, pero los cambios en la estructura de la distribución del ingreso también. Además, que existe una convergencia entre el crecimiento económico y el desarrollo, y "un factor esencial para lograr este mayor desarrollo es el mejoramiento de las condiciones y niveles de educación, salud, demografía, pobreza y desigualdad, debido a que una mejora en estas dimensiones no solo produce efectos directos sobre el desarrollo sino también efectos indirectos a través de mayores tasas de crecimiento y viceversa ${ }^{7} . "$

Por ende, la mayor concentración del ingreso en una ciudad indica que los frutos del crecimiento no benefician primordialmente a los más rezagados. En este sentido, otra de las características de la economía local son las desigualdades en la distribución del ingreso. Según la Misión de Estudio de la Extrema Pobreza (Mesep), el coeficiente de Gini en Cartagena subió a 0.489 en 2011, luego de estar en 0.38 en 1995.

Si bien la tasa de desempleo ha experimentado una notable caída si se compara con el periodo de la poscrisis económica de finales del siglo XX, y se haya registrado una reducción de la pobreza global y extrema en Cartagena, lo cierto es que en 2012, según la encuesta de Cartagena cómo vamos, esta última registró un preocupante aumento. Más de 15 mil cartageneros que entre 2008 y 2011 salieron de la pobreza extrema, volvieron a caer en ella. Al cierre de 2012 la cifra total de pobres extremos -gente que pasó hambrefue de 55 mil personas.

Otros datos muestran que Cartagena, entre los años 2000 y 2007, fue la ciudad donde más creció el nivel de vida (medido aproximadamente a través del PIB por habitante), pero también donde más se concentró el ingreso y crecieron las desigualdades en el ingreso. En efecto, el PIB por habitante creció casi 50\% en los ocho años mencionados (pasó de \$ 4.7 millones en 2000 a $\$ 7.1$ millones en 2007), mientras que la diferencia entre los ingresos de las personas terminó siendo de 36 veces.

Si se entiende por exclusión una situación de desventaja económica, profesional, política o de estatus social, producida por la dificultad que una persona o grupo tiene para integrarse a algunos de los sistemas de funcionamiento social, entonces se puede afirmar que: la exclusión en Cartagena es actualmente un fenómeno social y una cuestión política, económicamente mala, socialmente corrosiva y políticamente explosiva. Es un fenómeno que obliga a los

${ }^{7}$ Bernal, Raquel y otros (2009). Desarrollo económico: retos y políticas públicas. Documentos CEDE, (1). 
${ }^{8}$ Arrieta, R. (2011, abril) Los retos de la política social en Cartagena y el Caribe colombiano. Cartagena. Ponencia presentada en el IX Encuentro Centroamericano y del Caribe: Políticas sociales y trabajo social. Universidad de Cartagena, Cartagena.

${ }^{9}$ Arroyo, Daniel, (2009). Políticas sociales, ideas para undebatenecesario. Buenos Aires: La crujía ediciones.

${ }^{10}$ North, Douglas. (1995). Instituciones, cambio institucional y desempeño económico. México: Fondo de Cultura Económica. gobiernos locales y a la sociedad en general a modificar la creencia tradicional que el crecimiento económico invariablemente llegará a los pobres y excluidos para mejorar sus condiciones de vida ${ }^{8}$.

\section{II.Desarrollo local y asociatividad}

egún Arroyo el desarrollo local supone otras cuestiones -además de pensar en el territorio- y la más importante es "entender el concepto $\checkmark$ de desarrollo como crecimiento con impacto social, es decir, aquella actividad económica que no solo dinamiza el crecimiento económico del lugar sino que, al mismo tiempo, mejora las condiciones de vida mediante una mejor distribución del excedente que genere mejoras en los ingresos de la población"".

En palabras de Arroyo (2009:58) "el objetivo es concebir una convergencia entre el desarrollo local y las políticas sociales a partir de una dinámica territorial propia". Por esta razón pensar en la asociatividad como estrategia o dispositivo integrador entre la política social y económica, no es una quimera, sino que por el contrario puede llegar a consolidarse como una alternativa importante para impulsar respuestas afectivas a demandas complejas que plantea la sociedad actual.

־ensar en el desarrollo local de una determinada sociedad, lleva implícito una reflexión que pasa por precisar los conceptos de desarrollo, desarrollo local, asociatividad, actores, solidaridad, competencia, conflicto; entre otras categorías que son muy revisadas en la literatura socio antropológica. En esta oportunidad no se hará un extenso recorrido por estos conceptos sino que solo se presentaran de manera precisa su comprensión para este análisis.

\section{La asociatividad como posibilidad para incentivar desarrollo socioeconómico inclusivo}

Este trabajo parte del supuesto que la asociatividad es un mecanismo que apoya el desarrollo local de las sociedades; y se convierte en un dispositivo en la medida que los agentes del desarrollo están plenamente convencidos de la necesidad de trabajar de manera conjunta para superar contextos de pobreza, exclusión y desigualdad social. Por tanto, es necesaria una política social capaz de proteger, restablecer y garantizar los derechos de todas las personas, en particular de quienes han sido excluidos de los frutos del progreso.

Para lo cual, en palabras de North ${ }^{10}$, es necesario que existan instituciones, entendidas como reglas de juego, que garanticen y posibiliten el desarrollo de alternativas asociativas. 
En este entendido, el liderazgo estatal en las políticas públicas es fundamental pero no exclusivo, se hace necesaria una nueva relación Estado-Sector Productivo-Academia y Sociedad, que exhorte a la solidaridad y la responsabilidad social y genere confianza y seguridad, en el trabajo conjunto y cofinanciado que asegure la apropiación colectiva y sostenibilidad a la política social de la ciudad, la región y el país.

Es aquí donde entran en juego las características de procesos asociativos como el caso que se referencia, que puede señalarse como un ejercicio que vincula a todos los actores del desarrollo a saber: gobierno, sector productivo, sector educativo y las propias comunidades locales, regionales y/o nacionales; que podrían articular intereses y metas para trabajar en beneficio de los propios ciudadanos, que han quedado al margen de los procesos que se han emprendido desde iniciativas públicas y/o privadas.

Pensar en reducir los niveles de pobreza y desigualdad en Cartagena, obliga necesariamente a pensar que es imperativo: "promover un sistema de desarrollo productivo local, mediante espacios de concertación de actores públicos y privados que permitan desconcentrar el desarrollo, redefinir el perfil del aparato productivo, promover la inserción en los mercados regionales e internacionales y consolidar la ciudad-región, lo cual pasa por la identificación de sectores estratégicos, la priorización de apuestas productivas y la identificación de la infraestructura necesaria". (Corredor, 2010:190)

Cabe anotar que en este ejercicio, la organización empresarial asociativa, se equipara al concepto de Red social, con la idea que este comenzó siendo un concepto operativo que usaron algunos antropólogos británicos para describir y definir estructuras sociales de considerable amplitud y grado de intimidad, que en cierta forma se pueden comparar con las familias extensas o clanes, pero que no tienen como único lazo de unión el parentesco, sino que también se nutren de otros vínculos.

El análisis de redes enfatiza las relaciones que conectan la posición social dentro de un sistema, y da una visión global de la estructura social y sus componentes. La organización de las redes sociales se constituye, de esta forma, en concepto central del análisis de las propiedades estructurales de las redes, en las cuales interactúan los actores. Pero, lógicamente, la acción de los sujetos solo se analiza por sus implicaciones individuales, fácilmente se olvida cual es el contexto social dentro del cual el actor está envuelto; por ello 
Mitchell, C. (1969) ciones urbanas. Análisis de relaciones personales en pueblos de Africa Central Zambia: Manchester University Press.

${ }^{12}$ Castells, Manuel (2000). La era de la información. Madrid. Alianza Editorial. no hay que desconocer que cuando un actor participa en un sistema social, involucra a otros actores, los cuales significan punto de referencia para él.

Así mismo, reiterar que en este análisis se entiende como grupo asociativo: "Un conjunto especifico de vínculos entre un conjunto definido de personas, con la propiedad de que las características de esos vínculos como un todo puede usarse para interpretar la conducta social de las personas implicadas ${ }^{11}$."

Y más específicamente como una empresa red, es decir "aquella forma específica de empresa cuyo sistema de medios está constituido por la intersección de segmentos autónomos de fines. Por consiguiente, los componentes de la red son tanto autónomos como dependientes de ella y pueden ser partes de otras redes y, por ello, de otros sistemas de recursos dirigidos a otros objetivos ${ }^{12}$."

Es en este sentido que la experiencia asociativa se convierte en una alternativa posible para promover procesos formativos, y de consolidación de la experiencia empresarial de las pymes y además, promover las oportunidades de inversión a la par de consolidar y ampliar la red empresarial mediante políticas de innovación, desarrollo tecnológico, ampliación y diversificación de los mercados, de tal forma que se mejore la competitividad, y se aporte a la generación de empleo e ingresos.

Especial atención requieren las micro y pequeñas unidades económicas, pues el tejido productivo de la ciudad se caracteriza por su alta participación y tienen una enorme aversión al riesgo, a la innovación y al cambio tecnológico. De ahí que sean las que presentan mayor riesgo de liquidación (Corredor, 2010)

Es evidente que la experiencia asociativa debe aprovecharse como una herramienta para formar, capacitar, fomentar e impulsar entre las empresas asociadas, y entre estas y el sector público y privado, el involucramiento en procesos sociales que los enfrente con las necesidades de su entorno y como tal, que les impulse a desarrollar iniciativas que los conecte realmente con el desarrollo no solo de sus unidades productivas sino con su entorno y su contexto local y regional.

Puesto que no se puede hacer parte de una sociedad local sin preguntarse cómo las capacidades particulares de sus actores se conjugan para mejorar la calidad de vida de todos sus habitantes, es preciso entender que el desarrollo local es: "Un proceso de crecimiento concertado de una sociedad territorialmente delimitada, dentro de un contexto histórico y político, regional y nacional, 
que genera bienestar para el conjunto de sus miembros, a partir de potenciar las capacidades instaladas territorialmente (recursos humanos, institucionales y económicos) de manera sustentable y justa en el plano cultural, social y económico de la vida de las familias de esa sociedad ${ }^{13} . "$

\section{Una experiencia para la reflexión: Programa de Desarrollo Empresarial Sectorial, PRODES.}

La Asociación Colombiana de Pequeñas y Medianas Industrias ACOPI seccional Bolívar, a partir de 1998 preparó la promoción del Programa de Desarrollo Empresarial Sectorial - PRODES-, respondiendo al interés señalado por el propio sector metalmecánico, que permitió implementar en la ciudad la primera experiencia asociativa.

Luego de un proceso de concertación entre empresarios pertenecientes al sector; se constituyo ASIMECAR el 25 de abril del año 2002, y se inscribió ante la Cámara de Comercio de Cartagena el 24 de mayo del mismo año como una organización sin ánimo de lucro; iniciando sus actividades un grupo de 20 empresas, las cuales, de acuerdo a la dinámica particular que desarrollaban, fueron reduciéndose primero a 13 y luego a 8 empresas

El propósito de crear ASIMECAR era desarrollar entre las empresas de un mismo sector un conjunto de actividades asociativas, que propendieran por el mejoramiento de la gestión, la calidad, la productividad y la competitividad, en el corto, mediano y largo plazo.

En este sentido, según la directora ejecutiva de ACOPI la estrategia buscaba "agrupar por ramas, subsectores o sectores productivos, empresas de similar tamaño y alto grado de afinidad, para que a partir de su propio diagnóstico individual y grupal, desarrollen en cada fase del programa acciones integrales que permitan mejorar la capacidad negociadora para enfrentar la competencia interna y/o la inserción en los mercados internacionales ${ }^{14}$."

El sector metalmecánico de Cartagena se convirtió entonces, en uno de los principales precursores de esta tendencia de la Pymes hacia la asociatividad sectorial, corroborando que la estrategia se constituye como una alternativa válida que procura la supervivencia de estas unidades económicas mediante la búsqueda de nuevos métodos de producción, adquisición de nueva tecnología, control de gastos, seguimiento de las funciones administrativas a objetivos y metas organizacionales.
${ }^{13} \mathrm{http}: / /$ www.fcp.uncu. edu.ar/upload/Desarrollo local y ajuste es trūcturat_üna_suma base_cero. $\bar{p} \mathrm{df}$

${ }^{4}$ Asociación Colombiana e Pequeñas Industrias - Acopr- Seccional Bolí rarticipativo sector me almecánico. Cartagena: ACOPI. 
${ }^{15}$ Serrano, C. $(2002)$ Pobreza, capital social y ciudadanía. Asesoría de Integración Social ciudadanía y Pobreza. [En línea]. Disponible soriasparaeldesarrollo $\mathrm{cl} /$ secciones/documentos/pobreza capital social y ciudāania.pdf [2011, 24 de octubre].
De la experiencia se puede destacar la realización de un diagnóstico participativo sobre la problemática del sector, con el cual se logró identificar y priorizar las dificultades para el diseño de propuestas de gestión que redundarán en el mejoramiento de la calidad, la productividad y la competitividad de las empresas. Así mismo, permitió que las empresas desarrollaran diversas actividades que apoyaron la consolidación del proceso asociativo; al mismo tiempo que lograron una mayor proyección del grupo a la ciudad. La mayor dificultad encontrada fue la falta de confianza en la sostenibilidad y el débil sentido de pertenencia con el sector.

\section{Características de la asociación}

Del total de empresas asociadas, el 75\% se clasificó -según la Cámara de Comercio de Cartagena- como pequeñas empresas y el 25\% restante como microempresas. El 58.4\% de estas empresas se dedicaban a la elaboración y reparación de piezas y accesorios que requiere el sector metalmecánico, el $16.65 \%$ se dedica a la comercialización de piezas fabricadas, un $16.65 \%$ presta servicios de ingeniería mecánica y el $8.3 \%$ restante realiza instalaciones y mantenimiento a las industrias del sector metalmecánico. Estas empresas son en su mayoría de carácter familiar, de donde surge el capital inicial para su constitución, es decir se sustentan en el patrimonio familiar, pero luego a partir de la consolidación de las mismas, se logra la financiación a partir de recursos propios.

En cuanto a la generación de empleo, las empresas asociadas generan 350 empleos directos, 450 indirectos, distribuidos en rangos de ocupación que oscilan de 1 hasta 33 empleados, siendo el rango de 11 a 20 empleos el más significativo con una participación del 58.4\%, seguido del de más de 21 empleados con la participación del 25\% de las respectivas empresas asociadas.

La motivación del grupo en cuanto aportar al proceso de consolidación de la Asociación tuvo que ver con el logro de unos objetivos instrumentales. Tal como lo plantea Serrano: "Si bien las personas no entran en relaciones sociales de tipo cooperativo por cálculos racionales respecto del beneficio que esperan obtener, tampoco lo hacen por sentimientos altruistas. La motivación a la participación tiene relación con una combinación de aspectos instrumentales, que se refieren a la expectativa de mejorar sus oportunidades y activos, y aspectos simbólicos y cognitivos, asociados a la necesidad de pertenencia y reconocimiento en el medio grupal ${ }^{15}$." 
En este sentido, estar vinculado al gremio y a la asociación les permitió organizarse operativa y administrativamente para hacer un frente común a las amenazas y oportunidades del contexto; dicha vinculación fue considerada por algunos actores como una forma de contribuir al desarrollo local y en especial al desarrollo y crecimiento de la pequeña y mediana empresa en la ciudad, pues según sus apreciaciones, el fortalecimiento de la misma permite garantizar la estructura económica de la ciudad en términos de generación de empleo y recursos económicos.

Es así como el compromiso social de las empresas pertenecientes a la asociación, según los actores, se suscribe a la generación de empleos, generando beneficios directos e indirectos a través de la vinculación de algunos miembros del núcleo familiar a servicios de salud, recreación y pensión.

En este orden de ideas, la participación de la Asociación en la ciudad es asumida por algunos actores como una participación económica-productiva, razón por la cual la articulación de esta, con proyectos de otro orden desarrollados en la ciudad es incipiente, por cuanto el interés en ese tiempo se centró en la organización, consolidación y proyección del mismo; aunque en el discurso se manifiesta la importancia de la participación de la Asociación en otros espacios no necesariamente económicos; no se presentan acciones concretas que materialicen o cristalicen tal intencionalidad.

Estar asociado permitió la circulación e intercambio permanente de información relacionada con la situación política, social y económica del contexto que pueden significar una amenaza u oportunidad para el sector, esta lectura interna y externa del contexto en el marco de una cultura asociativa garantiza la sumatoria de activos, fuerzas, recursos para estar preparados, competir bajo los criterios de calidad en el mercado y aumentar el poder de negociación e interlocución con el Estado para participar en programas y proyectos gubernamentales y no gubernamentales, dirigidos al fortalecimiento de este sector.

La estrategia permitió al pequeño y mediano empresario, diseñar estrategias para ser más competitivos en el mercado, tales estrategias se traducen en logros visibles como: establecimiento de convenios directos (asociación con otras instituciones y organizaciones) e indirectos (gremio de la pequeña industria y otras instituciones y organizaciones) entre la Asociación con entidades públicas y privadas de la ciudad destacados. Así mismo la Asociación aúna esfuerzos de apoyo con las universidades locales, para el desarrollo de 
investigaciones y programas de formación dirigidos al fortalecimiento de las mismas en diversa áreas de interés.

\section{A manera de conclusión}

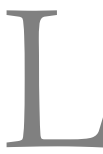

a red empresarial asociativa se asumió por los actores como mecanismos de integración entre empresas de un mismo sector que pretendían atender las amenazas del contexto a través de acciones conjuntas encaminadas a la productividad, calidad y competitividad. En este sentido, podría decirse que para los asociados la constitución y consolidación de esta no es un hecho espontáneo, sino que requiere de un propósito y de estrategias de inversión individuales y colectivas que implican obligaciones internalizadas y sentidas subjetivamente, las que se expresan en sentimientos y creencias respecto de los otros y que se instalan como normas y códigos de conducta. El grupo operó con unos objetivos a los que pueden denominarse instrumentales, pues responden a intereses particulares que benefician a las empresas y al colectivo en la medida que los fortalece frente a las amenazas del contexto.

Las empresas realizaron un ejercicio de común acuerdo de precios de mercado, para bienes y servicios ofrecidos por las empresas pertenecientes a la Asociación, y un proceso de mejoramiento de procesos de aseguramiento de los sistemas de calidad, razón por la cual la diferencia en cuanto a precios, oportunidad del servicio y calidad entre las mismas es mínima, mostrándose competitivos frente al mercado y al interior de las empresas.

En el afán de garantizar la supervivencia de las empresas se contempla a la Asociación como una acción colectiva de presión política para defender sus espacios y conquistar otros. Esta situación fue reconocida por los miembros de la Asociación en cuanto se constató que a través de la misma se lograron beneficios y participación en espacios a los que antes eran casi imposible de acceder.

No obstante, la participación en los aspectos políticos, sociales y económicos de la ciudad pareciera estar limitada en algunos actores, pues circunscriben la actuación de la Asociación a un espacio netamente económico, desconociendo que "...lo político no se restringe a los cauces formales de lo que coloquialmente

Ruiz, E. (1997). Minería democracia industrial, poder político y desa rrollo local en Río Tinto. Antropología, (13), 77 se entiende por política, sino que la acción del poder político, como una dimensión no aislada de los demás contextos y niveles de relaciones de poder, penetra la sociedad hasta sus resquicios más íntimos ${ }^{16}$." 
Es evidente que la Asociación acude a acciones de poder político para resignificar la participación de la PYME -en este caso del subsector metalmecánico- en el contexto local, trascendiendo la mera concepción que sobre política se tiene a partir de la participación en procesos sociales del trabajo en instituciones políticas o acciones de votación y/o contienda electoral.

Las características sociales tales como, estatus socioeconómico, sexo, formación académica, la edad y otros factores como la idiosincrasia y la historia personal subyacen a la dinámica de una red, es decir, influyen en el establecimiento de alianzas y oposiciones en un grupo social, así como también en los procesos interrelacionales suscitados en la misma, estos tal vez se constituyen en aspectos claves para tener presente en el análisis cualitativo de la red empresarial.

Entre las fortalezas identificadas por algunos miembros de este proceso asociativo se destacan: el poder de convocatoria del gremio, la red de relaciones de los actores que la integran y que están a disposición del grupo para la implementación de más y mejores proyectos y programas de fortalecimiento y crecimiento dirigido al sector, definición de unos objetivos, metas asociativas, la distribución de funciones, las competencias personales de los integrantes del mismo, la proximidad y antigüedad de las relaciones, el constante intercambio de información, el grado de conectividad y complementariedad de las actividades adelantadas, la existencia de una comunicación fluida, abierta, aunque para algunos este proceso está supeditado al grado de confianza entre los actores.

Estas particularidades del proceso de organización y consolidación de la asociación, dan cuenta de la importancia de la interrelación entre los diferentes actores de la sociedad local en Cartagena, lo cual, favorece el fortalecimiento del capital social de la región en beneficio del desarrollo económico, político, cultural y social de la ciudad.

Sin duda, y de acuerdo con los planteamientos de Arrieta (2011) se requiere de un impulso que recoja los esfuerzos de los actores del desarrollo e impulse: una política social capaz de proteger, restablecer y garantizar los derechos de todas las personas, en particular de quienes han sido excluidos de los frutos del progreso.

Para el caso de Cartagena, la asociatividad, en un marco político que propenda -a partir de su política económica y social- por la ampliación de oportunidades y la reducción de las inequidades; se convierte en un mecanismo para la superación 
de las trampas de pobreza, con políticas eficientes socialmente que privilegien la equidad en las oportunidades y el desarrollo de las capacidades, como elementos constitutivos de una vida digna y de una sociedad con justicia social.

El paradigma de que los frutos del crecimiento económico llegarán tarde o temprano a los más pobres y excluidos, no se puede seguir apoyando; se requiere de un proceso educativo que sensibilice a todos los sectores de la sociedad; en especial al sector empresarial, que por mucho tiempo ha considerado que su responsabilidad llega hasta la generación de empleo, este planteamiento está demostrado, ya no es real, no es suficiente. La sociedad requiere de la conciencia del profundo cambio en la perspectiva que sobre el desarrollo se ha tenido.

En este sentido, como afirma Arroyo (2009:59) es necesario que la política social y el desarrollo converjan, teniendo en cuenta la dinámica propia de cada territorio. Esto implica “...transformar la estructura territorial a favor de las actividades que generen valor agregado y procesos económicos que articulen regiones para formar cadenas y corredores productivos sólidos, para desarrollar una noción de políticas sociales inclusivas".

Las políticas sociales inclusivas además de ofrecer bienes y servicios básicos que aumentan las dotaciones iniciales de quienes se encuentran en peor situación en la sociedad, implican articular a la gente con lo productivo de tal manera que se puedan generar ingresos propios y promover capacidades productivas. Como afirma Corredor (2000:45), la política social también se asocia al empleo y a la distribución de ingresos, pues es necesario buscar soluciones a la problemática del desempleo, el subempleo y la informalidad. Para esto, se hace necesario la articulación público-privada que garantice la sostenibilidad de las políticas, la generación de oportunidades de empleo e ingreso y el avance en equidad.

Es la hora de la coparticipación, la solidaridad, la generación de confianza, y la disminución de la competencia desleal que ha generado verdaderos procesos de acumulación en beneficio de pocos que se han quedado con lo que muchos necesitan para tener mínimamente calidad de vida verdaderamente humana.

Se necesitan organizaciones empresariales implicadas en la participación activa en el diseño, implementación y evaluación de políticas sociales que contribuyan al cierre de la brecha que la exclusión y la desigualdad social, 
han generado en la sociedad. Aunque crecientemente la política social parece estar dirigida a los pobres, tenemos que recuperar una matriz mucho más amplia de esto que llamaríamos, en nuestro caso políticas socioeconómicas, que tienen que estar dirigidas a y definidas con la participación del conjunto de la sociedad.

\section{BIBLIOGRAFÍA}

Arrieta Flórez, Rosaura, (2011). Los retos de la política social en Cartagena y el Caribe colombiano. Cartagena: IX Encuentro Centroamericano y del Caribe: Políticas sociales y trabajo social. Universidad de Cartagena.

Arroyo, Daniel, (2009). Políticas sociales, ideas para un debate necesario. Buenos Aires: La crujía ediciones.

Asociación Colombiana de Pequeñas Industrias -ACOPI- Seccional Bolívar, (2002) Diagnóstico participativo sector metalmecánico. Cartagena: ACOPI.

Bernal, Raquel y otros, (2009). Desarrollo Económico: retos y políticas públicas. Documentos CEDE, número 1, Bogotá: Centro de Estudios sobre desarrollo Económico - Universidad de los Andes.

Castells, Manuel. (2000). La era de la información. Vol. 1-3. Madrid: Alianza Editorial.

Corredor Martínez, Consuelo. (2010). La política social en clave de derechos. Bogotá: Editorial Universidad Nacional de Colombia.

Escalera Reyes, J. y Ruiz, E. (1997). Minería, democracia industrial, poder político y desarrollo local en Río Tinto. Antropología, Número 13, pp. 77-102, Madrid: CIS editorial.

Mitchell, C. (1969) Redes sociales en situaciones urbanas. Análisis de relaciones personales en pueblos de África Central. Instituto de Estudios Africanos, Universidad de Zambia. Manchester University Press.

North, Douglas. (1995). Instituciones, cambio institucional y desempeño económico. México: Fondo de Cultura Económica.

Nupia, O. (2008). Documentos de política pública Piensa Colombia: los aportes de la academia. Colombia: Universidad Nacional de Colombia. En: http://www.nancypatricia.com/wp-content/ uploads/2009/12/Desarrollo-Economico.pdf Consultado el 24 de Octubre de 2011.

Payares Villegas, Z. (2003). Asociatividad empresarial. Estrategia para la competitividad. Bogotá: Fondo Editorial Nueva Empresa.

Rueda y Espinosa (2008). ¿Los pobres de hoy serán los pobres del mañana? Determinantes de la 
pobreza y la vulnerabilidad en Cartagena de Indias. Documentos de trabajo número 11. Cartagena: Universidad Tecnológica de Bolívar.

Serrano, C. (2002) Pobreza, capital social y ciudadanía. Asesoría para el Desarrollo. Red de Integración Social, Ciudadanía y Pobreza. En: http://www.asesoriasparaeldesarrollo.cl/secciones/ documentos/pobreza_capital_social_y_ciudadania.pdf Consultado el 24 de Octubre de 2011.

Vásquez-Barquero (1988). Desarrollo local una estrategia de creación de empleo. Madrid: Pirámide. 